\title{
STUDIES ON METHYL BROMIDE POISONING
}

\author{
Toshio KAKIZAKI \\ National Institute of Industrial Health, Kizuki-Sumiyoshi, Kawasaki
}

(Received May 15, 1967)

\begin{abstract}
An experimental study on acute methyl bromide poisoning was carried out in rabbits with subcutaneous injection of the oiled poison which was prepared by dissolving methyl bromide into olive oil.

Among the toxicological pictures caused by methyl bromide in rabbits paralysis of the hind limbs, leaving off drinking and reduction of urine seemed to be the characteristic signs of this poisoning.

Subcutaneous dosage of the oiled poison ( $50 \mathrm{mg}$ of methyl bromide per $\mathrm{kg}$ ) to rabbits resulted in a sharp elevation of blood free bromide level which indicated decomposition of methyl bromide, and a marked reduction of platelet count, blood serotonin level and blood water. After these changes free bromide level decreased very slowly in a long period of time, but the other three recovered to their values before dosage within 24 hours. From the fact that the toxicological pictures recovered with those three changing patterns, it was inferred that the toxic effects produced by the injected poison were due to methyl bromide itself and not to its decomposition products. Moreover there was a linear relationship between the dose amount and the net decrease in platelet count or blood water per cent at 5 hours after injection of methyl bromide.

Consequently the determinations of platelet count, blood serotonin level and blood water per cent were expected to be useful for the diagnosis of the methyl bromide poisoning in its early stages.
\end{abstract}

Methyl bromide has been widely used as a methylating agent in organic chemistry, as an insecticide and in fire extinguishers. Recently with the increase of fumigation works, methyl bromide poisoning has come into further notice. Owing to the lack of clear objective signs for the diagnosis of this poisoning, however, any definite abnormalities have not been revealed even in the clinical examinations of the poisoned patients. Accordingly it is urgent to find out some methods for the early detection and diagnosis of the poisoning.

This paper describes the hematological changes of rabbits brought about after injection of methyl bromide.

\section{Materials and Methods}

Male rabbits received methyl bromide $(20-120 \mathrm{mg} / \mathrm{kg})$ subcutaneously as an oiled poison which was prepared by dissolving liquid methyl bromide (Wako Pure Chemical Co.) into olive oil under low temperature. At various 


\section{T. KAKIZAKI}

times after injection of the oiled poison blood was collected from the heart using a plastic syringe attached with a siliconized needle into a plastic tube containing $1 / 10$ volume of 1.0 per cent $(\mathrm{w} / \mathrm{v})$ ethylenediamine tetraacetic acid disodium salt. The blood sampling should be carried out skillfully in order to avoid blood coagulation especially in the case of platelet counting.

\section{Determination of methyl bromide}

Methyl bromide in the blood was determined on total and free bromide. For the total bromide determination $1 \mathrm{ml}$ of blood was poured directly from a syringe into a tube containing $5 \mathrm{ml}$ of 5 per cent $(\mathrm{w} / \mathrm{v})$ alcoholic sodium hydroxide solution, shaken vigorously and stood for over 2 hours. For the free bromide determination another $1 \mathrm{ml}$ of blood was poured directly from a syringe into a tube cotaining $5 \mathrm{ml}$ of water, mixed gently and washed three times with $5 \mathrm{ml}$ of methylene chloride which was pretreated by the method of Shrader et al.1) Thus obtained each sample was transferred into each crucible and evaporated to dryness in an oven at $100^{\circ} \mathrm{C}$. Then the crucible was heated mildly on the Bunsen flame for about 10 minutes. One milliliter of water was added to the cooled crucible and the solution was slightly acidified with 50 per cent acetic acid solution and the bromide content was estimated by the microdiffusion analysis of Conway.2)

\section{Determination of serotonin and others}

After deproteination of $1 \mathrm{ml}$ of blood with $4 \mathrm{ml}$ of hot 10 per cent trichloroacetic acid solution the supernatant was washed twice with an equal volume of peroxide-free ether. In the case of platelet-poor plasma, deproteination was carried out by cold trichloroacetic acid solution. Then the later procedures were carried out by the method of Snyder et al.3)

In addition to the above determinations platelet count, hematocrit reading, plasma sodium- and potassium-ion content and blood water per cent were examined.

\section{Results}

The injection of methyl bromide to rabbits was followed by the outstanding symptoms such as paralysis of the hind limbs, depression of the respiration, decline of the heart rate, low body temperature, leaving off drinking and reduction of urine. Especially paralysis of the hind limbs, leaving off drinking and reduction of urine appeared to be characteristic signs of this poisoning.

In addition, the lethal dose of methyl bromide which was dissolved into olive oil was about $130 \mathrm{mg} / \mathrm{kg}$ of body weight in rabbits.

\section{Bromide levels in the blood}

The mean bromide levels in the blood of 4 rabbits before and after injec- 


\section{METHYL BROMIDE POISONING}

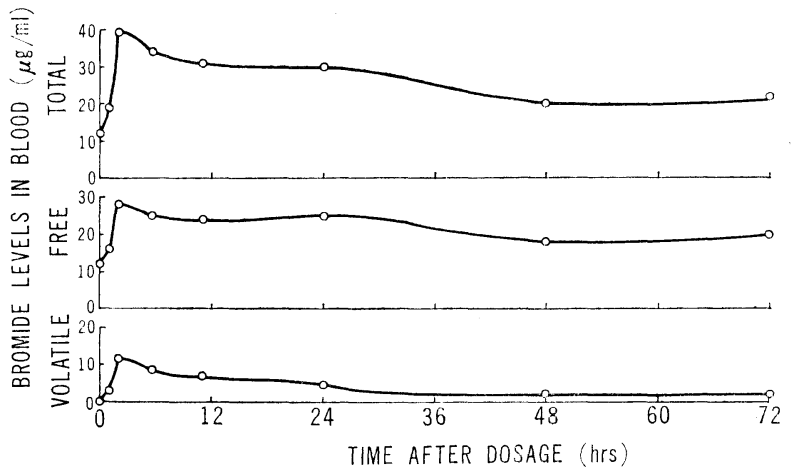

Fig. 1. Change in blood bromide levels of rabbits after methyl bromide injection. Each point shows the mean value of 4 rabbits. Rabbits received $50 \mathrm{mg}$ of methyl bromide per $\mathrm{kg}$ subcutaneously as an oiled poison which was prepared by dissolving liquid methyl bromide into olive oil. The volatile bromide level was taken from the difference between the levels of total and free bromide.

tion of $50 \mathrm{mg}$ of methyl bromide per $\mathrm{kg}$ of body weight were shown in Fig. 1. As seen in the figure the total bromide level in the blood showed a sharp increase after the injection of the poison giving the maximum value after 2 hours, then a very gradual return towards the level before treatment. The maximum value corresponded to about a half of the bromide level obtained by Clarke et al.4) in the case of frank methyl bromide poisoning. The free bromide level in the blood showed a parallel pattern to that of the total. The amount of volatile bromide which corresponded to the difference between the total and free bromide determinations changed similarly to these two bromide levels. From the pattern of the free bromide level in Fig. 1, it is supposed that methyl bromide injected is rapidly broken down to its components and liberated free bromide is eliminated slowly in a cosiderable period of time.

\section{Platelet counts and serotonin levels in the blood}

Judging from its symptomatic features such as paralysis of the hind limbs described above and from the high bromide content in the brain of a victim of methyl bromide poisoning as reported by Heimann,5) methyl bromide seemed to injure the central nervous system of animals. On the other hand, serotonin has been considered to play an important role in the functional regulation of the central nervous system and it is contained in platelets in the blood. The examination of serotonin and platelet level in the blood after methyl bromide injection, therefore, may give some informations on the toxic action of the poison.

Blood platelet count was achieved by the Fonio's direct procedure ${ }^{6)}$ within 


\section{T. KAKIZAKI}

30 minutes after blood collection. With methyl bromide injection the blood became so viscous and coagulable that a careful sampling of the blood was needed for platelet counting.

Methyl bromide injection $(50 \mathrm{mg} / \mathrm{kg}$ ) to 4 rabbits brought a marked decrease on both platelet count and serotonin level in the first 6 hours as shown in Fig. 2. In contrast with bromide level, however, these diminutions were recovered within 24 hours after dosage. Accordingly it is suggested that both the changes of platelet count and serotonin level were caused by methyl bromide itself and not caused by its decomposed components. Moreover the above mentioned symptoms appeared after injection of methyl bromide in this experiment seemed to correspond with the changing pattern of platelet count or serotonin level rather than with that of bromide level. This also suggests that the toxic effects produced by the injected poison were due to methyl bromide itself.

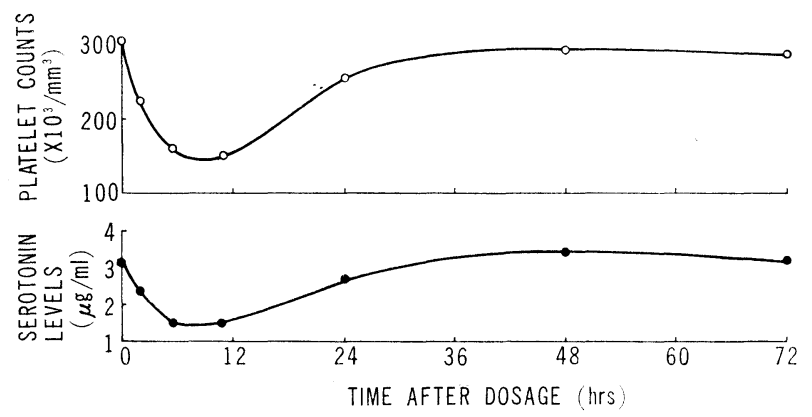

Fig. 2. Change in platelet counts and blood serotonin levels of rabbits after methyl bromide injection. Each point was obtained from the same blood samples as indicated in Fig. 1.

On the other hand it was expected that methyl bromide might release serotonin from platelet which ordinarily contains the most parts of serotonin in the blood. Serotonin level in the platelet-poor plasma, however, was very low and did not increase with the injection of the poison as tabulated in Table 1. It was assumed, therefore, that blood serotonin was held by platelet and its level reflected platelet count.

Table 1. Serotonin levels in the platelet-poor plasmas of rabbit $(\mu \mathrm{g} / \mathrm{ml})$ after dosage of methyl bromide $(50 \mathrm{mg} / \mathrm{kg})$.

\begin{tabular}{cccccc}
\hline Rabbit No. & \multicolumn{7}{c}{ Time after } & dosage (hrs) & & \\
& 0 & 2 & 5 & 11.5 & 24 \\
\hline 1 & 0.063 & 0.034 & 0.035 & 0.027 & 0.047 \\
2 & 0.110 & 0.100 & 0.087 & 0.085 & 0.072 \\
\hline
\end{tabular}

The experiments carried out with varying doses of methyl bromide to rabbits proved a linear relation between the dose amount and the net decrease 


\section{METHYL BROMIDE POISONING}

in platelet count at 5 hours after dosage as seen in Fig. 3. Such a proportionality as seen in platelet count was not clear in serotonin level, perhaps being due to mobilization of serotonin from some other sources into the blood in the case of a large dose of methyl bromide.
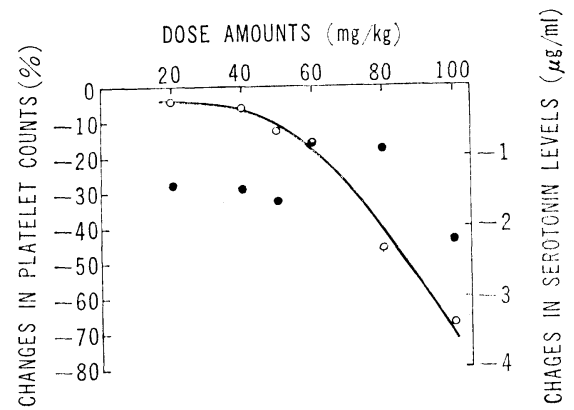

Fig. 3. Relationship between dose amounts of methyl bromide and changes in platelet counts or blood serotonin levels. Ordinate represents the net decrease in platelet counts (open points) and blood serotonin levels (closed points) respectively at 5 hours after dosage of the poison.

\section{Blood water per cent}

Leaving off drinking and reduction of urine observed in rabbits by methyl bromide injection suggested some changes of water maintenance in the body. As a means of the examination of water maintenance, blood water content was estimated with the method of weighing capillary tubes on the poisoned rabbits. As shown in Fig. 4, a marked decrease and a rapid recovery of blood water content were observed on 4 rabbits received methyl bromide $(50 \mathrm{mg} / \mathrm{kg}$ ). Its recovery to the level before injection was more earlier than those of platelet and serotonin level. As seen in Fig. 5 a linear relationship was also

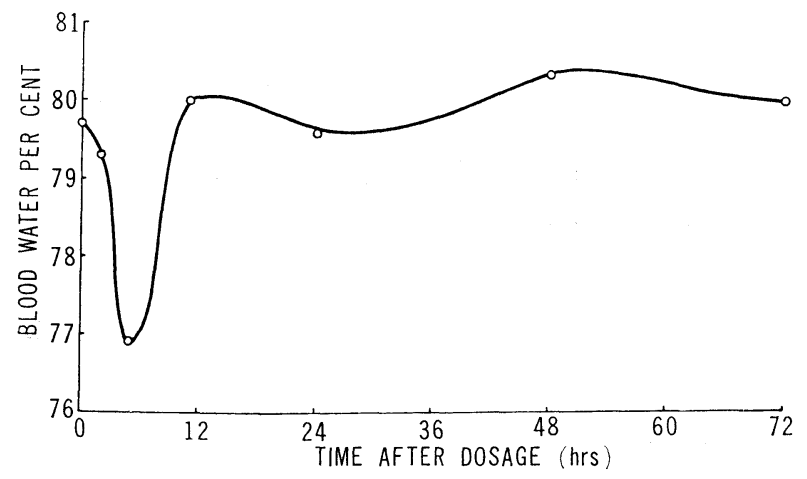

Fig. 4. Change in blood water per cent of rabbits after methyl bromide injection. Each point shows the mean value of 4 rabbits different from those indicated in Fig. 1. Experimental conditions were the same as those in Fig. 1. 


\section{T. KAKIZAKI}

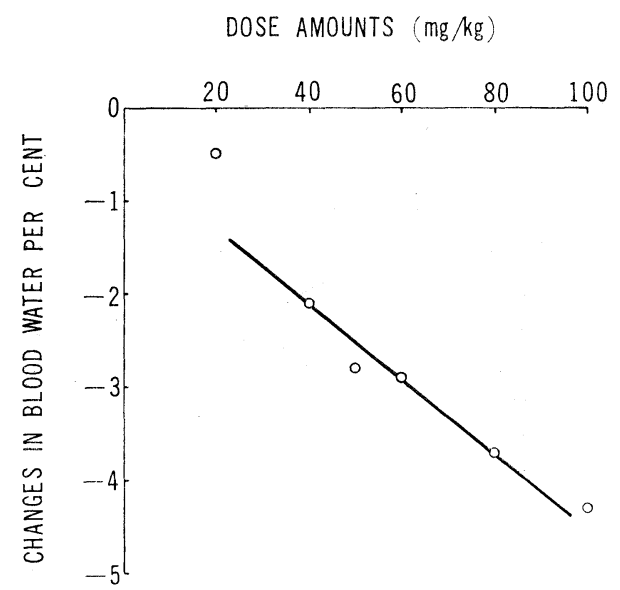

Fig. 5. Relationship between dose amounts and changes in blood water per cent. Ordinate represents the net decrease in blood water per cent at 5 hours after dosage of the poison.

obtained between the dose amount and the decreased amount of blood water at 5 hours after dosage.

On the other hand hematocrit reading of rabbits received methyl bromide showed rather fluctuation, but it decreased gradually after 24 hours. But plasma sodium- and potassium-ion content remained unchanged by injection of the poison to rabbits. From these results it may be supposed that methyl bromide provoked a hemoconcentration due to some disturbances of water maintenance in the animal bodies.

Table 2. Hematocrit readings (\%) of rabbits after methyl bromide injection. These results were obtained from the same animals as indicated in Fig. 1.

\begin{tabular}{cccccccccc}
\hline Rabbit No. & \multicolumn{7}{c}{ Time } & after & dosage (hrs) \\
& 0 & 1 & 2 & 5.5 & 11 & 24 & 48 & 72 \\
\hline 1 & 42.6 & 35.8 & 45.4 & 49.5 & 45.4 & 37.1 & 34.4 & 26.1 \\
2 & 44.0 & 48.1 & 46.8 & 46.8 & 49.5 & 38.5 & 30.2 & 28.9 \\
3 & 48.1 & 46.8 & 45.4 & 39.9 & 34.4 & 31.6 & 31.6 & 31.6 \\
4 & 41.4 & 39.9 & 35.8 & 33.0 & 30.3 & 27.5 & 27.5 & 26.1 \\
\hline
\end{tabular}

\section{Discussion}

In the present experiments methyl bromide was dissolved in olive oil and injected subcutaneously to rabbits in order to fix the dose amount and to make the experimental conditions uniform.

There may be some discrepancies in the toxic effects of methyl bromide between animals exposed to the poisonous gas and injected with the oiled 


\section{METHYL BROMIDE POISONING}

poison, since the metabolic process of the poison could be different in these two routes. For instance, rabbits exposed to the gas showed signs of salivation,7) irritation, restlessness and convulsions,8) while rabbits injected with the oiled poison showed the symptoms of leaving off drinking and reduction of urine as described above.

As to the reversible change of platelet count by injection of methyl bromide, judging from its sharp reversibility and decreasing extent it is difficult to assume that blood platelets were once broken down and then regenerated, or they were stored in the spleen for a while and then released into the blood. It is preferable to suppose that the platelet count change is due to a provisional aggregation of platelets and the subsequent trapping of aggregates in the small vessels as indicated in the studies of thrombosis by Gaarder et al.9) and Silver et al.10)

A sharp reversible change in blood water per cent was also brought about by methyl bromide injection as shown in Fig. 4. From the aspect of its sharp change, the change of blood water content may be attributable to a change in cell permeability of water rather than to a functional disorder of some organs in rabbits. On the other hand, prior to the platelet aggregation phenomenon above mentioned the platelet shape change towards a more spherical form was observed in human platelet-rich plasma by O'Brien.11) The shape change was accompanied with maximally a 26 per cent increase in volume which was not responsible for other than an explosive uptake of water12), being suggestive of a permeable change in platelet. Hence it seems that the platelet shape change is a predominant cause for the blood water change in the poisoned animals. This may be further supported by comparing both the changing patterns of platelet count and blood water per cent. However a more strict study will be required for this problem since plasma sodiumand potassium-ion content remained unchanged in the poisoned animals, which might be unfavourable for the above considerations suggesting water uptake by platelet.

At any rate the examination of platelet count, serotonin level and blood water content in the blood may be useful for the diagnosis of methyl bromide poisoning.

\section{REFERENCES}

1) Shrader, S. A., Beshgetoor, A. W. and Stenger, V. A. (1942). Ind. Eng. Chem. Anal. $E d ., 14,1$.

2) Conway, E. J. (1957). Microdiffusion Analysis and Volumetric Error, p. 306. Crosby Lockwood, London.

3) Snyder, S. H., Axelrod, J. and Zweig, M. (1965). Biochem. Pharmacol., 14, 831.

4) Clarke, C. A., Roworth, C. G. and Holling, H. E. (1945). Brit. J. Ind. Med., 2, 17.

5) Heimann, H. (1944). N. Y. St. Ind. Bull., 23, 103. 


\section{T. KAKIZAKI}

6) Fonio, A. (1952). Acta Haematol., 8, 363.

7) Beyne and Goett, (1934). Arch. Méd. Pharm. Navales., 124, 409.

8) Duvoir, M., Fabre, R. and Layani, F. (1939). Bull. Soc. Pharmacol., 46, 15.

9) Gaarder, A., Jonsen, J. and Laland, S. (1961). Nature, 192, 531.

10) Silver, M. D., Stehbens, W. E. and Silver, M. M. (1965). Nature, 205, 91.

11) O'Brien, J. R. (1965). Nature, 207, 306.

12) Bull, B. S. and Zucker, M. B. (1965). Proc. Soc. Exptl. Biol. Med., 120, 296. 